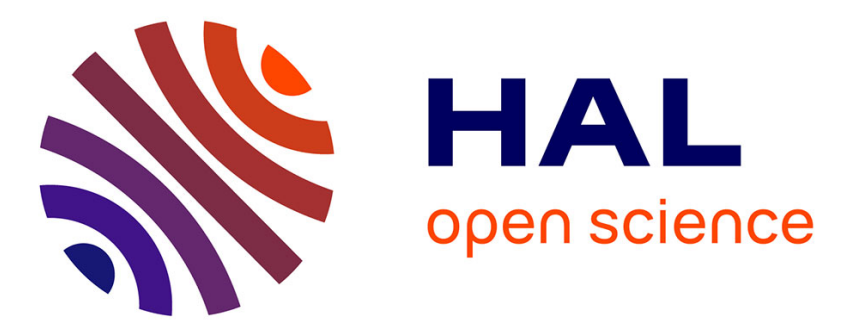

\title{
Internal 3D Printing of Intricate Structures
}

Théophane Nicolas, Ronan Gaugne, Cédric Tavernier, Valérie Gouranton, Bruno Arnaldi

\section{To cite this version:}

Théophane Nicolas, Ronan Gaugne, Cédric Tavernier, Valérie Gouranton, Bruno Arnaldi. Internal 3D Printing of Intricate Structures. 6th International Conference on Culturage Heritage - EuroMed 2016, Oct 2016, Nicosia, Cyprus. pp.432-441. hal-01391762

\section{HAL Id: hal-01391762 https://hal.science/hal-01391762}

Submitted on 3 Nov 2016

HAL is a multi-disciplinary open access archive for the deposit and dissemination of scientific research documents, whether they are published or not. The documents may come from teaching and research institutions in France or abroad, or from public or private research centers.
L'archive ouverte pluridisciplinaire HAL, est destinée au dépôt et à la diffusion de documents scientifiques de niveau recherche, publiés ou non, émanant des établissements d'enseignement et de recherche français ou étrangers, des laboratoires publics ou privés. 


\title{
Internal 3D Printing of Intricate Structures
}

\author{
Théophane Nicolas ${ }^{1}$ Ronan Gaugne ${ }^{2}$ Cédric Tavernier ${ }^{3}$ Valérie Gouranton ${ }^{4}$ and Bruno \\ Arnaldi $^{4}$ \\ ${ }^{1}$ Inrap, France \\ ${ }^{2}$ Université de Rennes 1/IRISA-Inria Rennes, France \\ ${ }^{3}$ Image ET, France \\ ${ }^{4}$ INSA de Rennes/IRISA-Inria Rennes, France
}

\begin{abstract}
Additive technologies are increasingly used in Cultural Heritage process, for example in order to reproduce, complete, study or exhibit artefacts. 3D copies are based on digitization techniques such as laser scan or photogrammetry. In this case, the $3 \mathrm{~d}$ copy remains limited to the external surface of objects. Medical images based digitization such as MRI or CT scan are also increasingly used in $\mathrm{CH}$ as they provide information on the internal structure of archaeological material. Different previous works illustrated the interest of combining 3D printing and CT scan in order to extract concealed artefacts from larger archaeological material. The method was based on 3D segmentation techniques within volume data obtained by CT scan to isolate nested objects. This approach was useful to perform a digital extraction, but in some case it is also interesting to observe the internal spatial organization of an intricate object in order to understand its production process. We propose a method for the representation of a complex internal structure based on a combination of CT scan and emerging 3D printing techniques mixing colored and transparent parts. This method was successfully applied to visualize the interior of a funeral urn and is currently applied on a set of tools agglomerated in a gangue of corrosion.
\end{abstract}

Keywords: Archaeology, Tomography, 3D printing.

\section{$1 \quad$ Introduction}

Cultural Heritage professionals such as archaeologists and conservators regularly experience the problem of working on concealed artefacts and face the potential destruction of source material without real understanding of internal structure or state of decay. For example, artefacts may be encased in corroded materials or a block of ash, or integrated with, and inseparable from, larger assemblies, such as manufactured objects composed of several pieces.

The work presented in this paper aims to develop a non-destructive workflow for analysing and documenting the internal structure of artefacts, improving diagnostic techniques and knowledge by combining medical imaging technologies such as Computed Tomography (CT) with 3D printing. Our methodology allows to create digital 
and physical 3D surrogates of objects to investigate, analyse and interpret their internal structure through volumetric scanning, 3D image rendering, and 3D printing.

Combinations of $\mathrm{CT}$ scan and 3D printing technologies have already been proposed in $\mathrm{CH}$ contexts. The projects presented in [1] and [2] both propose the use of 3D printers, combined with $\mathrm{CT}$ images, to reproduce high-value pieces, Chinese chess pieces in the first case, and Gold jewels in the second case. In [10], copies of fragile bones are used for sharing during study process and for exhibition.

This methodology was also successfully applied to physically access to encased artefacts through 3D images, and 3D printed replications without any irreversible physical action on the original material [3], even in the case of a disaggregated artefact [4]. In particular, in the first work, a removable copy of a Gallic weight illustrates how this technique allowed to better understand the internal structure of an artefact. This is also the case in the work of McKnight et al. [5] where a bones assembly from an animal mummy has been reconstituted for scientific analysis and public exhibition purpose. In both [6] and [2], a 3D print of the original shape of the archaeological material composed of an aggregate of several objects is proposed. This kind of production is useful to study the shape of disaggregated container.

In addition to these different works, it can also be interesting to have a view of both the external and internal structure of archaeological material in order to understand its production process, or to spatially localize the different notable internal items. The goal of the work presented in this paper is to propose a tangible representation of a complex intricate structure through advanced 3D printing. The process is applied to a funeral urn, and combines $\mathrm{CT}$ scan, 3D model processing, and 3D printing.

\section{Archaeological context}

The excavation of the site of Domaine de la Bizaie in Guipry (Brittany, France, Fig. 1, Left) in a preventive archaeological context uncovered a trapezoidal shaped funerary enclosure. The central area housed ten cremation burials containing pottery vessels from the Iron Age (Excavation L. Aubry, Inrap, [7]). The exceptional state of preservation of some of these cremations prompted us to use tomography to analyse their contents. It allowed to highlight a number of metal objects as in the F42A cremation (Fig. 1, Right). 


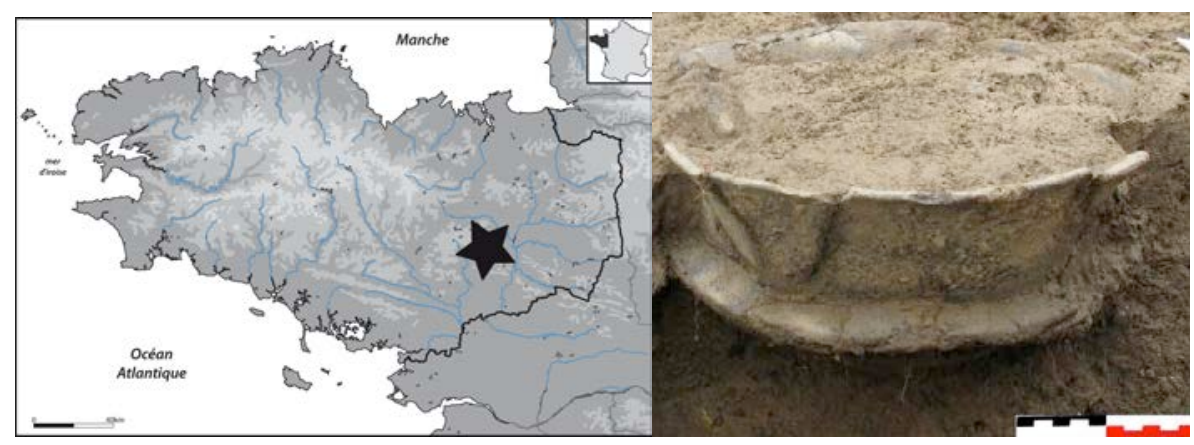

Fig. 1. Left: Domaine de la Bizaie, Guipry, France. Right: The F42 cremation, in situ

\section{Description of the work}

The process used in this study is based on a combination of CT scan, 3D processing and $3 \mathrm{D}$ printing. The main goal of the process is to physically display the internal structure of the archaeological material. We thus chose to explore two possibilities offered by $3 \mathrm{D}$ printing: physical copy of a part of the initial material and global printing with transparency. The process follows these different steps:

1. A computed tomodensitometry of the archaeological material was performed generating a database of X-Ray images, with density data.

2. Surface 3D meshes are generated from the previous database. Each mesh corresponds to a density range.

3. The 3D models are processed to fit 3D printing constraints.

4. The resulting $3 \mathrm{D}$ models are $3 \mathrm{D}$ printed.

\subsection{Technical Environment}

CT scan: The CT scanner used in this study is a Siemens SOMATOM sensation 16 owned by the French company Image ET (http://www.image-et.fr/) who delivers services and expertise on X-Ray images and associated tools.

The three-dimensional explorations were performed through two passes (acquisition) and 2D/3D images post-processing. The two acquisitions were a topogram (or radio mode) for positioning the slices to be realized and a helix scan.

The scan generates a dataset under DICOM format [8] of 512x512 pixels 2D slice images.

3D data processing. The data generated during the $\mathrm{CT}$ scan was processed with the free software Osirix, an image processing application for Mac dedicated to DICOM images. The $3 \mathrm{D}$ surface rendering functionality was used to generate $3 \mathrm{D}$ meshes cor- 
responding to different density ranges. This functionality allows to generate the surface of a set of points whose density value is within a defined range.

The 3D model was then manually processed in order to remove unwanted data and automatically processed in order to get a ready-to print file.

3D printing. We performed two different tasks of $3 \mathrm{D}$ printing on the urn, using two technologies: 3D printing of an internal artefact, and 3D printing of a whole material with transparency. The first 3D printer was a Stratasys Mojo owned by our institute. This 3D printer uses Plastic Jet Printing to print objects from ABS, in layers as thin as $178 \mu$, with a maximum dimension of $12.7 \mathrm{~cm} \times 12.7 \mathrm{~cm} \times 12.7 \mathrm{~cm}$. For the second printing, transparent 3D printing for complex objects appeared to be a not very developed technique. We contacted several Companies delivering advanced 3D printing services and only one, the CADindus Company (www.cadindus.fr), positively answered to our request. It uses a technology of multimaterial and multicolor additive manufacturing by resin polymerization for the production of the copies, on a Stratasys Objet500 Connex3 3D printer. This printer has a printing capacity of $49 \mathrm{~cm} \times 35 \mathrm{~cm} \times$ $25 \mathrm{~cm}$, with an accuracy of $30 \mu$.

\subsection{Application of the process to the urn}

Scan of the urn. The urn was scanned as a whole at one time. As the sediments inside the urn were not too dense, the scan was performed with the values of $120 \mathrm{kV}$ and 350 mAs. In order to scan the entire urn, the field of view was sized to $320 \mathrm{~mm} \times 320 \mathrm{~mm}$, resulting in a resolution of $625 \mu$. We worked in an extended Hounsfield scale (from 10.000 to +40.000 ), in order to distinguish between fragments of bones and metallic parts and get a finer view of the metallic objects. A volume rendering is presented in Fig. 2.

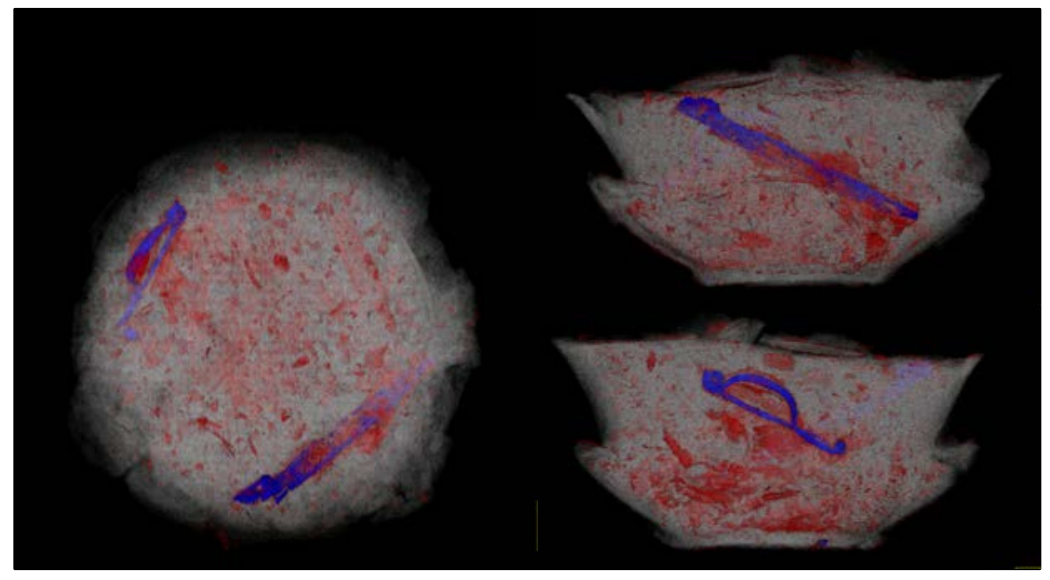

Fig. 2. Volume rendering of the urn 
The three views of volume rendering of the urn presented in Fig. 2 highlight the metal parts in blue, with two notable objects, a fibula and a knife blade. The red parts are fragments of bones. The sediments are displayed in transparent grey.

3D data generation and processing. Three meshes were generated from the data with the Osirix software (Fig. 3). The first mesh, for the metallic parts, corresponds to the points whose radio-density is between 4500 and 10950, the second mesh, for the urn shape and sediments, between 700 and 1300, and the last mesh, for the bones parts, between 1600 and 2300 .
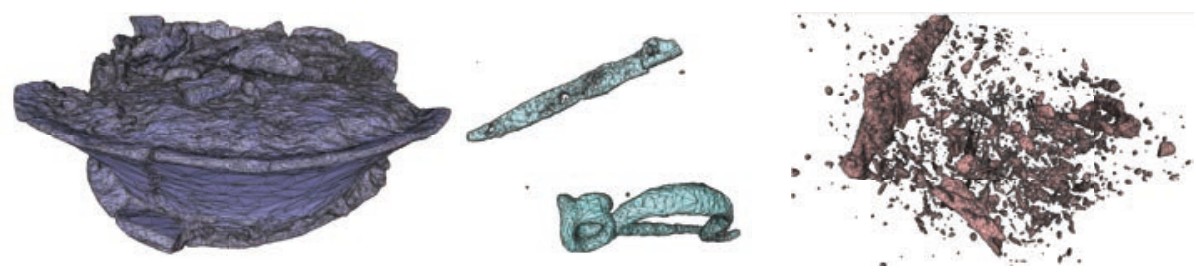

Fig. 3. Left: Meshes for the urn and sediments, Middle: metallic parts, Right: bones

Two issues arose after the generation of the meshes. First, corrosion gangues on the knife blade and fibula (Fig. 4, left) had a radio-density within the range of bones, visible in the mesh of the bones (Fig. 3, right). These gangues were easily removed as they were completely separated from other bones. Second, the mesh of the urn and ashes contained many galleries and cavities due to worms and insects (Fig. 4, right). All the galleries had to be manually removed and closed in order to get a clean transparency inside the urn. This task took several weeks, with many exchanges with the archaeologists in order to validate the modifications of the model.

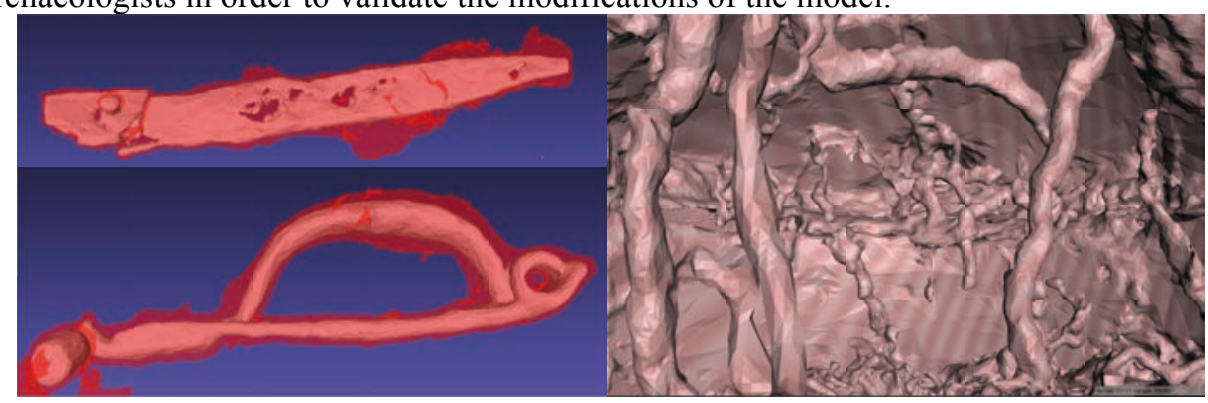

Fig. 4. Left: Gangue of corrosion for the metallic parts. Right: Worms and insect galleries inside the urn

The targeted impression technique for the full urn required filling the volume of the urn, with a negative print of the metallic objects and bones fragments. This task was performed with 3D Boolean operations in a 3D modeling tool. 
Printing of the fibula. The mesh of the fibula was isolated in order to propose a physical virtual extraction of this object whose shape appeared to be very well preserved. The process of producing the physical copy of the fibula from the digital data appeared to be very simple and fast (less than one day). The resulting copy of the fibula is presented in Fig. 5.

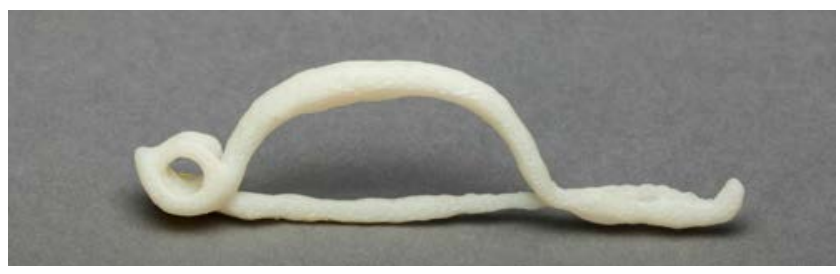

Fig. 5. 3D printing of the fibula

Printing of the urn. In order to keep close to the initial volume renderings from tomography (Fig. 2), we chose to render the bones in magenta and the metallic parts in cyan. The printing phase took 50 hours and required $8 \mathrm{~kg}$ of matter. It was printed with successive layers, bottom up (Fig. 6 left). The resulting printed object was covered with a pink support matter (Fig. 6 right)

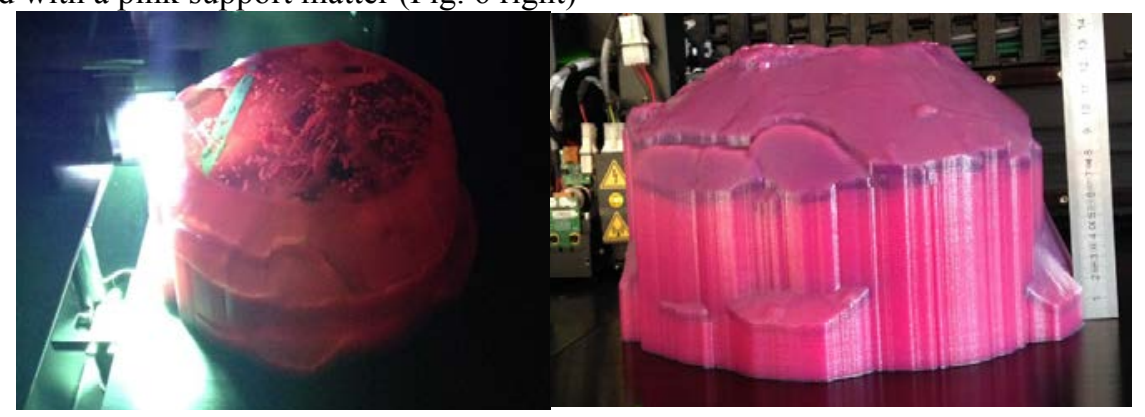

Fig. 6. Left: On-going 3D printing of the urn. Right: The printed urn with its support matter

Post-processing. An important post-processing of the object produced by the 3D printer is required to obtain a good transparency rendering (Fig.7). This post processing, which lasted one week, consisted in four phases: (i) Removal of the support matter, (ii) Photo-bleaching during $72 \mathrm{~h}$ at 6500 kelvins (« day light »), (iii) Sanding cycles with different size grit (from 180 to 800 ), (iv) Coating in sterile conditions. 


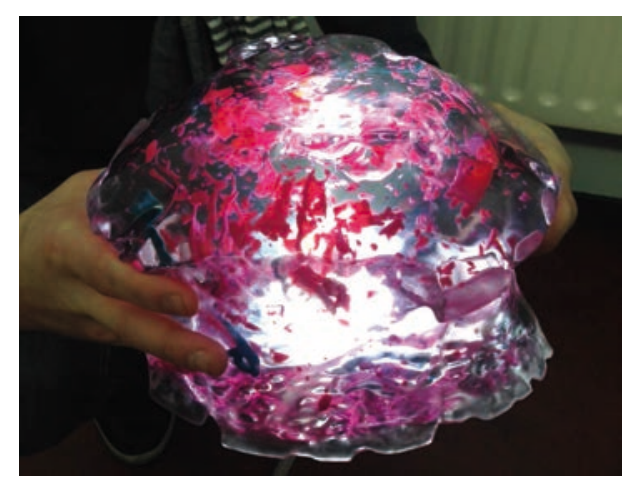

Fig. 7. Transparency view in the 3D printed urn

\section{$4 \quad$ Discussions on the results}

Before its actual excavation, the cremation $42 \mathrm{~A}$ was the subject of a CT and a $3 \mathrm{D}$ reconstruction. $3 \mathrm{D}$ volume rendering informs on the preservation of the burial, the urn and the cremated bone block (its fragmentation and the deformations of the internal masses bioturbations ...). It allows the identification, location and orientation in space of each artifact in a sustainable manner, the visualization and localization of the bone mass in its entirety and the observation of the nature of the sediment's components in the urn. These elements offer the possibility of taking immediate precautionary measures before any manual intervention. Furthermore, the digitization permits operations of "virtual" manipulation of artifacts to increase the observation providing the first interpretations before excavation. It also serves as supporting tangible "evidence" for elements that are difficult to characterize in 2D. With the segmentation tools, it is possible to obtain an independent 3D model of an artifact that can be virtually manipulated before its excavation. The additive techniques allow tangible handling and initial observations before the provision of the original. This process allows the provision of information within hours, which is not the case for the "operational chain" commonly implemented that can take months if protective measures are implemented, prior to the study of the archaeological material, as presented in Fig.8.

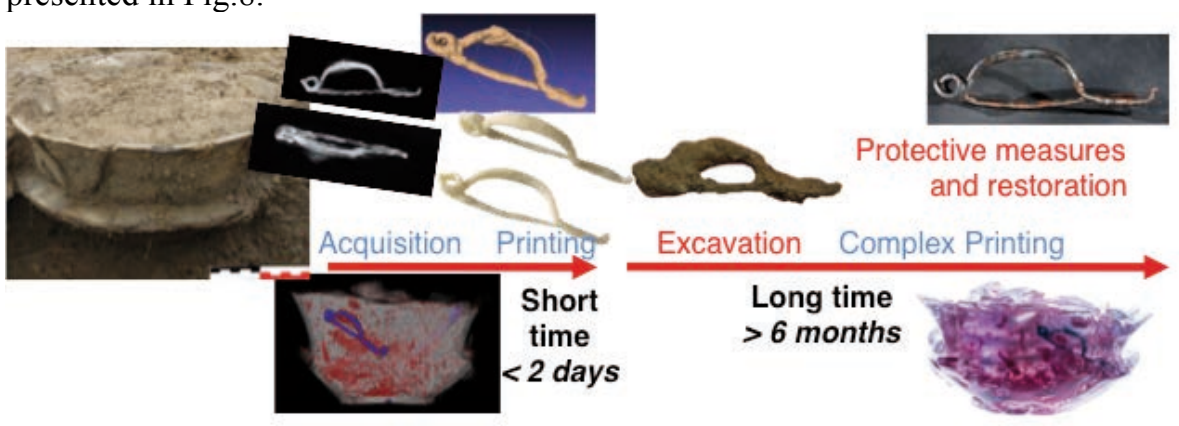

Fig. 8. Timelines of digital (blue) and physical (red) excavations 
The CT scan provided information on the structural state of the object, and allowed the identification of the cluster type of the fibula. Nevertheless, the observation of 2D sections and $3 \mathrm{D}$ reconstruction did not make possible to truly characterize the number of turns of the spring and the pin holder. Different segmentations were performed resulting in a restitution of the object with an approximate resolution of the details, which requires a systematic return to the real object in fine. This model has been the subject of a 3D impression (Fig. 9 left) that allowed to corroborate a number of observations from CT scans but also to confirm with certainty the number of turns of the spring and the shape of the. Also of note is the tangible nature of 3D printing with respect to the digital model. These comments have all been confirmed after the implementation of protective measures (Fig. 9 right and Fig. 10 left). The characterization of the initial surface condition during manual cleaning by a conservator revealed a setting of longitudinal striations on the arc (Fig. 10 right). Only this manual intervention may allow detection of such a setting very difficult to determine virtually on a small object.
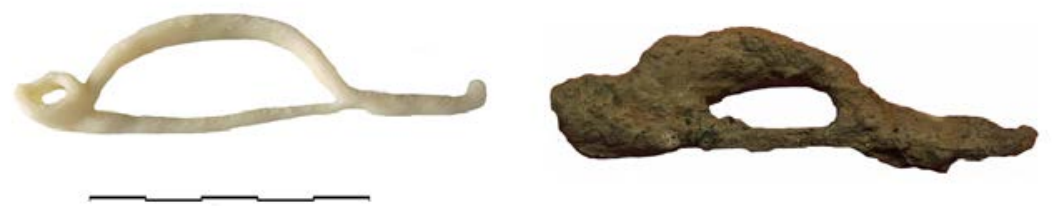

Fig. 9. Left: the 3D printed fibula Right: the real fibula, after its excavation, and before the restoration process.

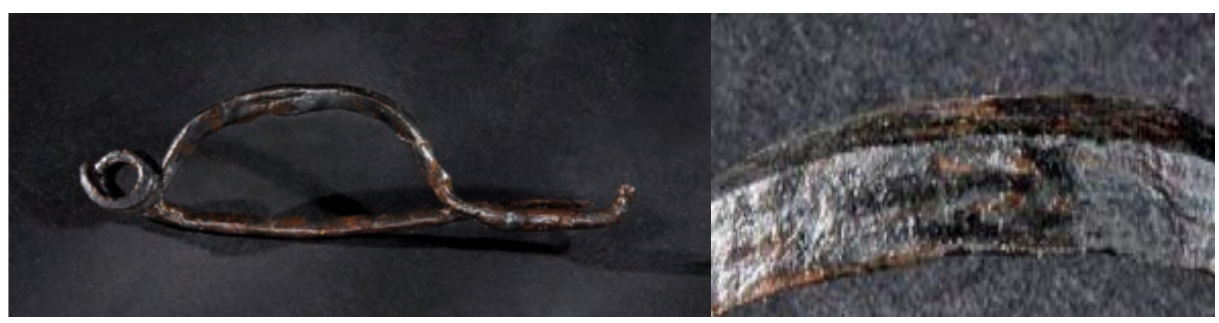

Fig. 10. Left: The fibula after restoration. Right: Details of the etched patterns on the fibula

The CT scan reveals the different elements using an arbitrary color application (bone remains in red, metal in blue, biological indices in grey, etc.). For the neophyte, this palette can disturb, but is quickly understood. For the anthropologist, while ordinarily the information is supplied layer by layer in stratigraphic excavation, this comprehensive $3 \mathrm{D}$ visualization is more interactive. Translating from $2 \mathrm{D}$ to $3 \mathrm{D}$, is none other than the transition from virtual to real and requires each expert to integrate this type of imaging. The complex 3D printing digital model overcomes this difficulty. After treatment, the printing transparency provides a 3D print of the container and contents (here funeral-deposit burned bones and iron objects knife rivet and fibula, Fig. 11). 
Printing on both its tangible nature allows "direct" access to information and the physical handling of cremation deposit. It allows viewing and manipulation of the vessel and its contents in full and with integrity. Printing allows visualization of burned bones that are imperceptible when excavating (too small, fragile, or appearing negative, to be taken), but also some well-preserved bones (at least here, a long bone) and the determination of iron artefacts (here released from their mineralization). 3D printing also provides information for the reconstruction of the funerary urn. This tangible medium allows a manipulation / simple visualization to work on the analysis (such as the distribution of artifacts and the burial gestures) but also as a support for the excavation [2]. Indeed, 3D printing is the only tangible medium of context preserved after the excavation of the incineration.

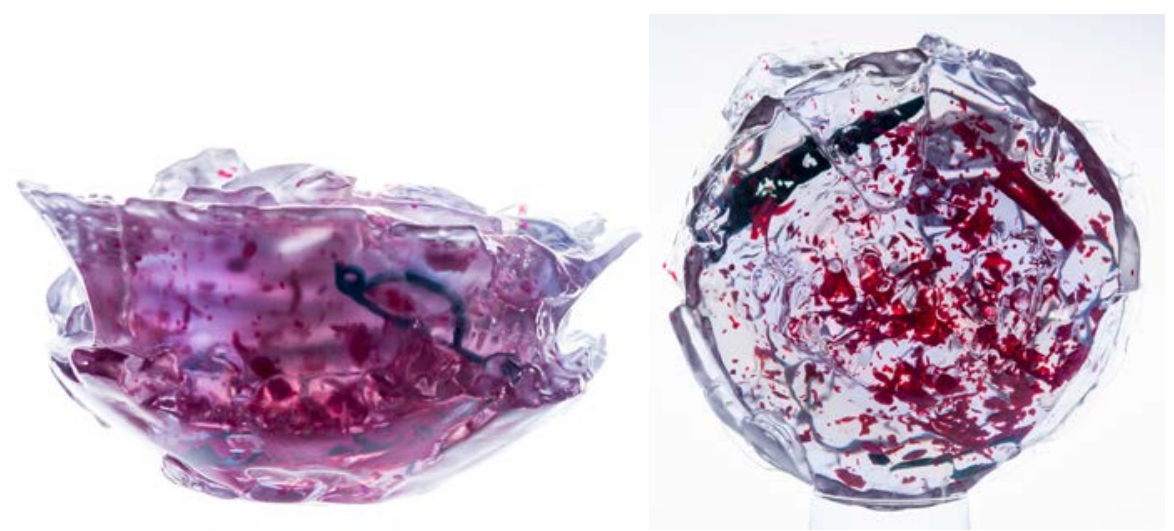

Fig. 11. Front and bottom views of the $3 \mathrm{D}$ printed urn

\section{$5 \quad$ Conclusion and future works}

For the archaeologist, the first asset of the scanner remains the immediacy of information, essential in the case of diagnostics where time is short. Well before the excavation of a container (funeral vase...), imaging allows the establishment of a protocol, the scheduling of specialists' intervention, the possible solicitation of a conservator curator to keep the fluidity of the scientific treatment of the remains [9].

In this process, the substitution of the original by certified copies can be attractive for a valuation framework, given the fragility of unstable materials to exhibit. The archaeological sensitive materials suffer during traveling. Accidents due to manipulation (packaging, transport and installations) are inevitable. 3D printing offers promising perspectives regarding the diversity of materials faithful to the look, the weight, the texture of the original... From the image to the copy of an original, it takes an average a half-day with an easy correction of the design since we can re-intervene in the template file at any time. We also underline its importance in an educational set- 
ting with teaching kits embellished with 3D restitutions subject to multiple manipulations, but also for people with disabilities.

The processes used open up new exploratory research. For example, the possibility of obtaining a model of old or recent bioturbations (earthworms galleries ...) in a funerary urn provides information on the presence of a perishable container and 3D printing of a model provides access to "all" of the skeletal remains.

\section{Aknowledgement}

This project was funded by the CNRS Imag'In IRMA project, France.

\section{References}

1. S. D. Laycock, G. D. Bell, N. Corps, D. B. Mortimore, G. Cox, S. May, and I. Finkel. 2015. Using a Combination of Micro--Computed Tomography, CAD and 3D Printing Techniques to Reconstruct Incomplete 19th-Century Cantonese Chess Pieces. J. Comput. Cult. Herit. 7, 4, Article 25 (February 2015)

2. H. Haßmann, T. Heintges, B. Rasink, S. Winghart,F.-W. Wulf, Der bronzezeitliche Hortfund von Gessel, Stadt Syke, Landkreis Diepholz, Berichte zur Denkmalpflege in Niedersachsen Veröffentlichung des Niedersächsischen Landesamtes für Denkmalpflege Hameln : Niemeyer, Vol. 32, No. 1, 2012, p. 23-28

3. Nicolas T., Gaugne R., Tavernier C., Gouranton V., Arnaldi B. Preservative Approach to Study Encased Archaeological Artefacts. Int. Conf. on Culturage Heritage, LNCS, Vol.8740. pp.332-341, 2014.

4. Nicolas T., Gaugne R., Tavernier C., Petit Q., Gouranton V., Arnaldi B., Touching and interacting with inaccessible cultural heritage, in Presence: Teleoperators and Virtual Environments, MIT Press, 2015, 24 (3).

5. L.M. McKnight, J.E. Adams, A. Chamberlain, S.D. Atherton-Woolham, R.J. Bibb, Application of clinical imaging and 3D printing to the identification of anomalies in an ancient Egyptian animal mummy. J. of Archaeological Science: Reports, 3, 2015,pp. 328 - 332.

6. T. Nicolas, R. Gaugne, C. Tavernier, V. Gouranton, B. Arnaldi, « Tomography and 3D printing for the study of archaeological artefacts » in proceedings of the conference « New technologies for cultural heritage », Cambridge Scholars Publishing, to appear.

7. Aubry L., Le Puil-Texier M., Un enclos funéraire de l'âge du Fer, Domaine de La Bizaie, Guipry, (Ille-et-Vilaine) : rapport de fouilles, Cesson-Sévigné : Inrap GO, 113-119, 2014.

8. Mustra, M., Delac, K., Grgic, M.: Overview of the DICOM Standard. ELMAR. 50th International Symposium. pp. 39-44, 2008.

9. M. Biron, S. Hurtin, T. Nicolas, C. Tavernier, La tomographie des objets archéologiques complexes et /ou altérés : outil d'identification, d'analyse et d'aide à la décision pour les mesures conservatoires, In Restaurer l'ordinaire. Exposer l'extraordinaire, Journée des Restaurateurs en Archéologie (JRA), Arles 2014

10. V. Mitsopoulou, D. Michailidis, E. Theodorou, S. Isidorou, S. Roussiakis, T. Vasilopoulos, S. Polydoras, G. Kaisarlis, V. Spitas, E. Stathopoulou, C. Provatidis, G.Theodorou, Digitizing, modelling and 3D printing of skeletal digital models of Palaeoloxodon tiliensis (Tilos, Dodecanese, Greece), Quaternary International 379, 2015 